Provided for non-commercial research and education use. Not for reproduction, distribution or commercial use.

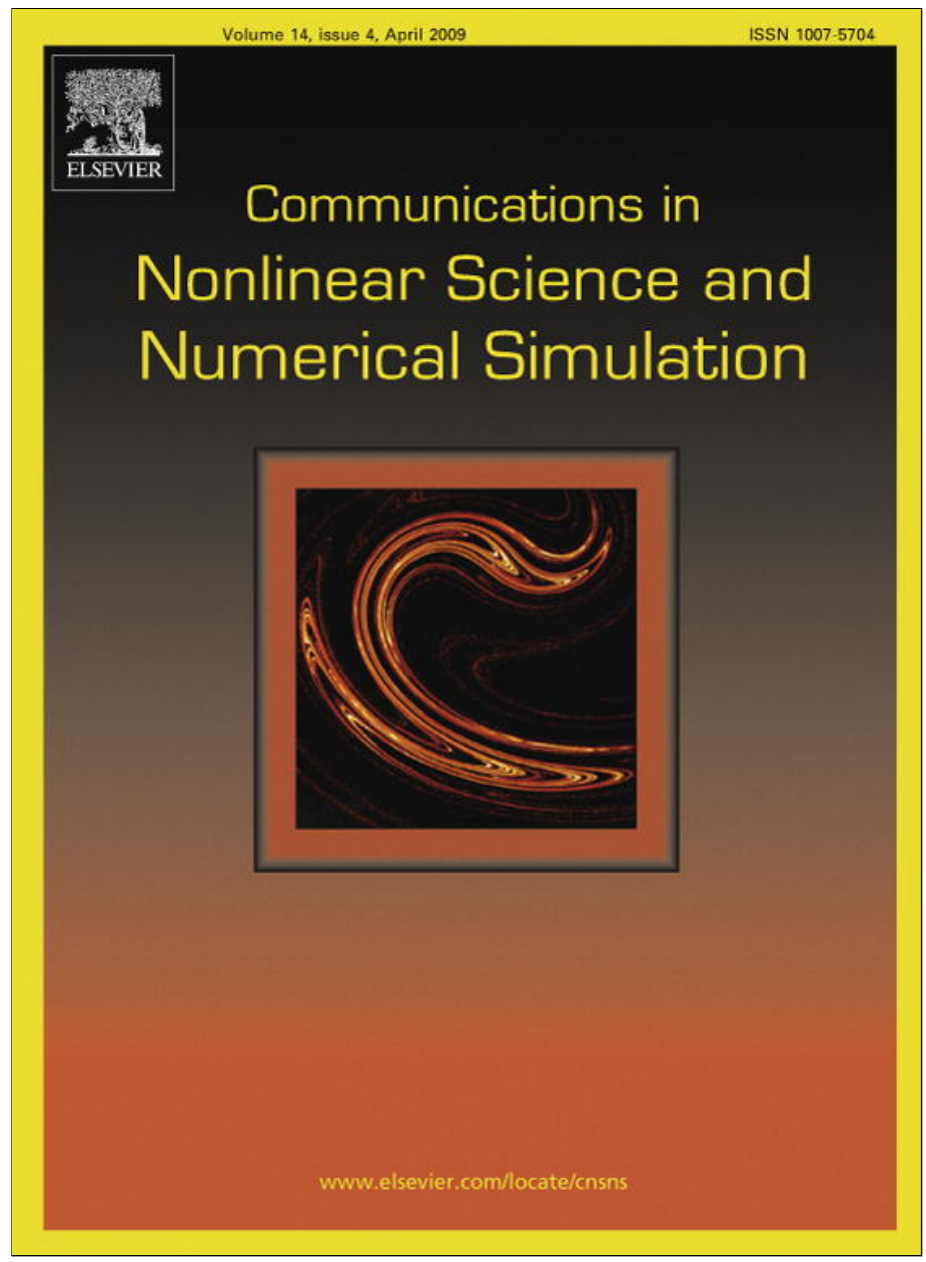

This article appeared in a journal published by Elsevier. The attached copy is furnished to the author for internal non-commercial research and education use, including for instruction at the authors institution and sharing with colleagues.

Other uses, including reproduction and distribution, or selling or licensing copies, or posting to personal, institutional or third party websites are prohibited.

In most cases authors are permitted to post their version of the article (e.g. in Word or Tex form) to their personal website or institutional repository. Authors requiring further information regarding Elsevier's archiving and manuscript policies are encouraged to visit:

http://www.elsevier.com/copyright 


\title{
Numerical study of the influence of diffusion of magnetic particles on equilibrium shapes of a free magnetic fluid surface
}

\author{
Sergey Beresnev ${ }^{\mathrm{a}}$, Viktor Polevikov ${ }^{\mathrm{b}}$, Lutz Tobiska ${ }^{\mathrm{a}, *}$ \\ a Institut für Analysis und Numerik, Otto-von-Guericke-Universität Magdeburg, Postfach 4120, D-39016 Magdeburg, Germany \\ ${ }^{\mathrm{b}}$ Department of Computational Mathematics, Belarusian State University, Independence Avenue 4, 220030 Minsk, Belarus
}

\section{A R T I C L E I N F O}

\section{Article history:}

Received 14 February 2008

Accepted 6 April 2008

Available online 14 April 2008

\section{PACS:}

75.50.Mm

$02.60 . \mathrm{Cb}$

47.65.Cb

Keywords:

Magnetic liquid

Free surface

Numerical simulation

\begin{abstract}
A B S T R A C T
A mathematical model and a computational method for studying the influence of the particle diffusion on equilibrium shapes of a magnetic liquid is developed. It is then applied on the ferrohydrostatic problem of doubly connected equilibrium shapes of a magnetic fluid located on a horizontal plate around a vertical cylindrical conductor with a direct current. Numerical simulations show the limits of the uniform concentration approximation.
\end{abstract}

(c) 2008 Elsevier B.V. All rights reserved.

\section{Introduction}

Because of their ability for ponderomotive interaction with an external magnetic field, magnetic fluids have not only provoked the development of a new direction in fluid mechanics but have become a new technological material which found a wide application in engineering [1-4]. A magnetic fluid is a stable colloidal suspension of ferromagnetic particles in a carrier liquid (oil, water, bio-compatible liquid). The size of particles is of the order of $10^{-8} \mathrm{~m}$, and they are in the Brownian motion state in the carrier liquid. Owing to the fact that the particles possess magnetic properties, not only Brownian motion but also a magnetophoresis diffusion process takes place in a magnetic fluid [1,5]. This diffusion process becomes significant when the magnetic fluid is under the influence of a high-gradient magnetic field.

The subject of the present study is the classical ferrohydrostatic problem on doubly connected equilibrium shapes of a magnetic fluid located on a horizontal plate around a vertical cylindrical conductor with a direct current $[1,2,6]$. Axisymmetric free-surface shapes which are realizing under the influence of the magnetic field of the conductor are preferable for the mathematical model because of the magnetic field structure. Assuming a linear magnetization law and neglecting the capillary pressure jump on the surface, the problem has been solved analytically, see [1,2]. The numerical solution for the more detailed problem of taking both the capillary jump but also the (non-linear) Langevin's magnetization law into consideration, is realized in [6]. However, it should be emphasized that the simplest theoretical models studied in [1,2] and the more advanced in [6] are both based on the assumption of homogeneity of the magnetic fluid, i.e. the effect of magnetophoresis of ferromagnetic particles in the fluid has been completely neglected. The purpose of this work is the investigation of the

\footnotetext{
* Corresponding author. Tel.: +49 391 6718650; fax: +49 3916718073.

E-mail addresses: sergey.beresnev@mathematik.uni-magdeburg.de (S. Beresnev), polevikov@bsu.by (V. Polevikov), tobiska@mathematik.uni-magdeburg.de (L. Tobiska).
} 
influence of diffusion of magnetic particles on equilibrium axisymmetric shapes of the free magnetic fluid surface. As the fluid magnetization value is directly proportional to the particle concentration in the fluid volume $[1,2,7,8]$, which is determined by the magnetic field structure, the diffusion effect is expected to become appreciable under a strongly non-uniform magnetic field.

\section{Mathematical model}

\subsection{General equations}

Under the assumption that the magnetic particles are of spherical form and of equal size, the magnetic particle mass transfer in a magnetic fluid can be described by the equation [7,8]:

$$
\begin{aligned}
& \frac{\partial C}{\partial t}+\mathbf{v} \cdot \nabla C=D \nabla \cdot(\nabla C-C L(\xi H) \nabla(\xi H)-C \eta \mathbf{g}) \\
& \xi=\frac{\mu_{0} m_{\mathrm{m}}}{k T}, \quad \eta=\frac{m}{k T}
\end{aligned}
$$

where $C$ is the volume concentration of the particles in the colloid; $t$ the time variable; $\mathbf{v}$ the velocity of a convective motion; $D$ the diffusion coefficient; $H$ the magnetic field intensity; $g$ the acceleration of gravity; $\mu_{0}=4 \pi \times 10^{-7} \mathrm{H} \mathrm{m}^{-1}$ is the magnetic constant (magnetic permeability of vacuum); $m_{\mathrm{m}}$ the magnetic moment of a particle; $k=1.3806568 \times 10^{-23} \mathrm{~J} \mathrm{~K}^{-1}$ the Boltzmann constant; $T$ the particle temperature; $m$ the mass of a particle; and

$$
L(\gamma)=\operatorname{coth} \gamma-\frac{1}{\gamma}
$$

the Langevin function. We assume that the fluid is incompressible and the boundary is impermeable, thus

$$
\operatorname{div} \mathbf{v}=0, \quad \mathbf{v} \cdot n=0
$$

where $n$ denotes the outer unit normal at the boundary.

Eq. (1) is supplemented with the condition of impermeability of boundaries by particles:

$$
\frac{\partial C}{\partial n}-C \xi L(\xi H) \frac{\partial H}{\partial n}-C \eta g_{\mathrm{n}}=0
$$

where $g_{n}$ is the normal component of the gravity acceleration.

A uniform distribution of concentration at the initial state,

$$
C=C_{0}=\text { const. }, \quad t=0
$$

is assumed. Eq. (1) together with conditions (2) and (3) represent the mathematical model of the diffusion process of ferromagnetic particles in a magnetic fluid. Notice that the solution of problem (1)-(3) satisfies the condition of conservation of the mean concentration:

$$
\frac{1}{V} \int_{V} C \mathrm{~d} V=C_{0} \text { for all } t \geqslant 0
$$

where $V$ is the fluid volume (or the spatial domain of definition of the problem).

For $t \rightarrow \infty$, we obtain the steady-state concentration problem with $\mathbf{v}=0$ which can be written in the form

$$
\begin{aligned}
& \left\{\begin{array}{l}
\nabla \cdot(\nabla C-C \nabla(\ln \varphi))=0 \quad-\quad \text { inside the fluid } \\
\frac{\partial C}{\partial n}-\frac{\partial(\ln \varphi)}{\partial n} C=0 \quad-\quad \text { at the boundary } \\
\int_{V} C \mathrm{~d} V=C_{0} V
\end{array}\right. \\
& \varphi=\exp \left(\int_{0}^{\xi H} L(\gamma) \mathrm{d} \gamma+\eta \mathbf{g} \cdot \mathbf{r}\right)=\frac{\sinh (\xi H)}{\xi H} \exp (\eta \mathbf{g} \cdot \mathbf{r})
\end{aligned}
$$

where $\mathbf{r}$ is the radius-vector of the current space point.

As shown in [8], problem (4) admits an analytical solution:

$$
C=\varphi \frac{C_{0} V}{J_{0}}, \quad J_{0}=\int_{V} \varphi \mathrm{d} V
$$

Estimations of the order of magnitude show that the influence of the gravity force on the diffusion of Brownian particles is negligible, which simplifies the formula for determining $\varphi$ :

$$
\varphi=\varphi(\xi H)=\frac{\sinh (\xi H)}{\xi H}
$$

In this case, isolines of the magnetic field strength are also isolines of the particle concentration. 
The magnetic properties of the magnetic fluid are determined by its magnetization $M$ which depends on both the magnetic field intensity $H$ and the particle concentration $C$. In ferrohydrodynamics $[1,2,7,8]$, the Langevin's magnetization law for a non-uniformly concentrated magnetic fluid is defined by the formula

$$
M=M(H, C)=\frac{M_{\mathrm{s}}}{C_{0}} L(\xi H) C
$$

where $M_{\mathrm{s}}$ is the magnetic fluid saturation magnetization; $C_{0}$ the mean concentration corresponding to a uniform distribution of the particles.

Equilibrium shapes of a free magnetic fluid surface are described by the Young-Laplace equation. In the static case, it takes the form

$$
\sigma K=\rho \mathbf{g} \cdot \mathbf{r}+\frac{1}{2} \mu_{0}\left(\frac{M}{H} H_{\mathrm{n}}\right)^{2}+\mu_{0} \int_{0}^{H} M \mathrm{~d} H+p_{\mathrm{f}}-p_{0}
$$

where $K$ is the sum of principal free-surface curvatures which is positive if the surface is convex; $\sigma$ the surface tension coefficient; $\rho$ the fluid density; $p_{0}$ the pressure in a surrounding non-magnetic medium; $p_{\mathrm{f}}$ is the thermodynamic pressure in the fluid.

Eq. (8) is supplemented with boundary conditions as well as with the non-local (integral) condition of fluid volume conservation. As boundary conditions we understand either conditions where the fluid contacts a solid wall specified by the wall geometry and a given wetting angle, or symmetry conditions.

\subsection{Statement of the problem}

Consider a drop of a magnetic fluid located on the horizontal plate around a vertical cylindrical conductor (see Fig. 1). Let $R_{0}$ be the radius of the conductor, $V$ be the fluid volume, $\alpha$ be the wetting (contact) angle on the solid walls. As the free surface is supposed to be axisymmetric, its shape is determined by the equilibrium meridian line. We introduce cylindrical coordinates $R, Z$ by bringing the $Z$-axis into coincidence with the symmetry axis, directing it opposite to the gravity vector, and placing the $R$-axis in the plate surface. Let $S$ be the arc length of the unknown meridian line, that takes the value $S=0$ at the upper contact point, i.e. at $R=R_{0}$, and $S=\ell$ at the lower contact point, i.e. at $Z=0$. The meridian shape will be described by the parametric functions $R(S), Z(S)$. Then, the surface curvature is defined by the formula $K=-\left(R Z^{\prime}\right)^{\prime} /\left(R R^{\prime}\right)$ where the prime stands for differentiation with respect to $S$.

Direct current generates an azimuthal magnetic field of intensity $H=I /(2 \pi R)$ around the conductor where $I$ is the current strength. Owing to the azimuthal orientation of the field, its structure does not depend on the magnetic properties of the fluid. In addition, since $H_{\mathrm{n}}=0$ at each point of the axisymmetric free surface, the magnetic pressure jump at the surface equals zero.

By using (5)-(7), it is not difficult to show that

$$
\int_{0}^{H} M \mathrm{~d} H=\frac{M_{\mathrm{s}} V}{\xi J_{0}}(\varphi-1), \quad J_{0}=\int_{V} \varphi \mathrm{d} V=2 \pi \int_{0}^{\ell} \varphi Z R R^{\prime} \mathrm{d} S
$$

where $\varphi=\varphi(R)=\sinh (\xi H) /(\xi H), H=H(R)=I /(2 \pi R), R=R(S), Z=Z(S)$. Consequently, Eq. (8) is transformed into

$$
Z^{\prime \prime}=R^{\prime} F, \quad 0<S<\ell
$$

with the abbreviation

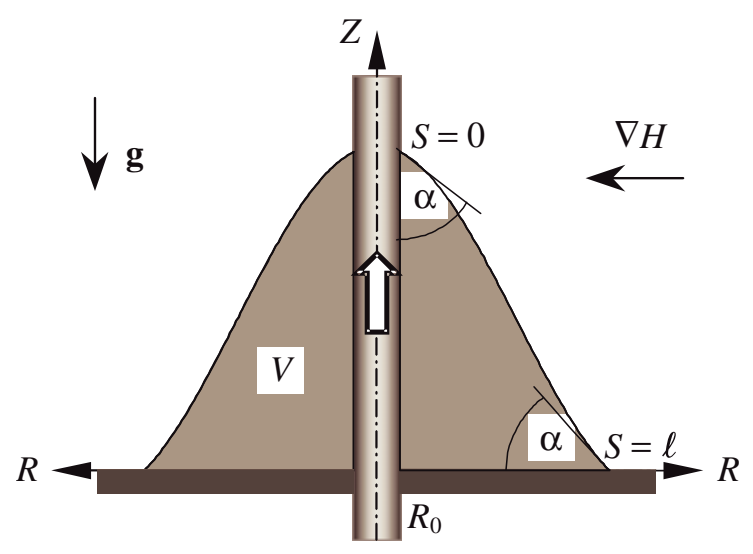

Fig. 1. Illustration of the problem. 


$$
F=\frac{\rho g}{\sigma} Z-\frac{\mu_{0} M_{\mathrm{s}} V}{\sigma \xi J_{0}} \varphi-\frac{Z^{\prime}}{R}+\text { const. }
$$

An arc length parametrization has been used, thus differentiating the identity $\left(Z^{\prime}\right)^{2}+\left(R^{\prime}\right)^{2}=1$ with respect to $S$ results in a second differential equation:

$$
R^{\prime \prime}=-Z^{\prime} F
$$

Natural conditions at the contact points are the following

$$
\begin{aligned}
& R(0)=R_{0}, \quad Z^{\prime}(0)=-\cos \alpha, \quad R^{\prime}(0)=\sin \alpha \\
& Z(\ell)=0, \quad Z^{\prime}(\ell)=-\sin \alpha, \quad R^{\prime}(\ell)=\cos \alpha
\end{aligned}
$$

Assuming that the drop volume $V$ is a prescribed quantity, we can determine it as the volume of a body of revolution:

$$
V=2 \pi \int_{0}^{\ell} Z R R^{\prime} \mathrm{d} S
$$

Thus, the mathematical statement of determining the free surface shape in parametric representation $(R(S), Z(S)), 0 \leqslant S \leqslant \ell$, consists of the differential equations (9) and (10), the boundary conditions (11), and the integral constraint (12).

Now, we introduce the dimensionless variables

$$
s=\frac{S}{R_{0}}, \quad z=\frac{Z}{R_{0}}, \quad r=\frac{R}{R_{0}}
$$

and reformulate problem (9)-(12) in dimensionless form

$$
\left.\begin{array}{l}
z^{\prime \prime}=r^{\prime} f, \quad r^{\prime \prime}=-z^{\prime} f, \quad 0<s<L \\
r(0)=1, \quad z^{\prime}(0)=-\cos \alpha, \quad r^{\prime}(0)=\sin \alpha \\
z(L)=0, \quad z^{\prime}(L)=-\sin \alpha, \quad r^{\prime}(L)=\cos \alpha \\
U=2 \pi \int_{0}^{L} z r r^{\prime} \mathrm{d} s
\end{array}\right\}
$$

where the following abbreviations have been used

$$
\begin{aligned}
& f=B o z-\frac{A_{1}}{J_{1}} U \varphi-\frac{z^{\prime}}{r}+\gamma, \quad \varphi=\varphi(r)=\frac{r}{A_{2}} \sinh \frac{A_{2}}{r} \\
& J_{1}=2 \pi \int_{0}^{L} z r r^{\prime} \varphi \mathrm{d} s, \quad U=\frac{V}{R_{0}^{3}} \\
& B o=\frac{\rho g R_{0}^{2}}{\sigma}, \quad A_{1}=\frac{\mu_{0} M_{s} R_{0}}{\sigma \xi}, \quad A_{2}=\frac{\xi I}{2 \pi R_{0}}
\end{aligned}
$$

Note that $L=\ell / R_{0}$ and $\gamma$ are undefined constants, whereas Bo (Bond number), $A_{1}, A_{2}$, and $U$ (dimensionless volume) are given dimensionless parameters. In order to determine the constant $\gamma$, we write the first of the Eq. (13) in the form

$$
\left(r z^{\prime}\right)^{\prime}=r r^{\prime}\left(B o z-\frac{A_{1}}{J_{1}} U \varphi+\gamma\right)
$$

and integrate it from $s=0$ to $L$. Taking into consideration the boundary conditions and the non-local constraint (last equation in (13)), we obtain a formula defining the constant $\gamma$ :

$$
\begin{aligned}
& \gamma=\frac{2}{r^{2}(L)-1}\left(\cos \alpha-r(L) \sin \alpha-\frac{B o U}{2 \pi}+\frac{A_{1} U J_{2}}{J_{1}}\right) \\
& J_{2}=\int_{0}^{L} r r^{\prime} \varphi \mathrm{d} s
\end{aligned}
$$

The solution $z(s), r(s)$ of the dimensionless problem (13) and (14) describes the equilibrium shape of the free surface and is determined by five parameters: $B o, A_{1}, A_{2}, U$ and $\alpha$.

\section{Computational algorithm}

Following the strategy in [9], we reformulate problem (13) and (14) in the new variables $\bar{s}=S / \ell=s / L \in[0,1]$, $\bar{z}=Z / \ell=z / L, \bar{r}=R / \ell=r / L$ for getting an explicit formula to compute the (unknown) constant $L$ in the iteration process. Moreover, we introduce the new unknown $\beta(\bar{s})$ being the angle between the tangent at the equilibrium line $(\bar{z}(\bar{s}), \bar{r}(\bar{s}))$, $0 \leqslant \bar{s} \leqslant 1$, and the $\bar{r}$-axis. Having in mind that $\bar{z}^{\prime}=\sin \beta$ and $\bar{r}^{\prime}=\cos \beta$, the problem (13) and (14) can be reformulated as

$$
\left.\begin{array}{l}
\beta^{\prime}=\Phi, \quad \beta(0)=\alpha-\frac{\pi}{2}, \quad \beta(1)=-\alpha, \\
\bar{z}^{\prime}=\sin \beta, \quad \bar{z}(1)=0, \quad \bar{r}^{\prime}=\cos \beta, \quad \bar{r}(0)=\frac{1}{L}
\end{array}\right\}
$$


where

$$
\begin{aligned}
& \Phi=\Phi(\beta, \bar{r}, \bar{z}, L)=B o \bar{z} L^{2}-\frac{A_{1} U}{I_{1} L^{2}} \varphi-\frac{\sin \beta}{\bar{r}}+\bar{\gamma} \\
& \varphi=\frac{\bar{r} L}{A_{2}} \sinh \frac{A_{2}}{\overline{\bar{r}} L}, \quad L=\left(\frac{U}{I_{0}}\right)^{1 / 3} \\
& \bar{\gamma}=\frac{2}{\bar{r}^{2}(1)-\bar{r}^{2}(0)}\left(\bar{r}(0) \cos \alpha-\bar{r}(1) \sin \alpha-\frac{B o U}{2 \pi L}+\frac{A_{1} U I_{2}}{I_{1} L^{2}}\right) \\
& I_{0}=2 \pi \int_{0}^{1} \bar{z} \bar{r} \cos \beta \mathrm{d} \bar{s}, \quad I_{1}=2 \pi \int_{0}^{1} \bar{z} \bar{r} \cos \beta \varphi \mathrm{d} \bar{s}, \quad I_{2}=\int_{0}^{1} \bar{r} \cos \beta \varphi \mathrm{d} \bar{s}
\end{aligned}
$$

We discretize the differential equation problem (15) on the uniform grid $\left\{\bar{s}_{i}=i h \mid i=0,1, \ldots, N, h=1 / N\right\}$ by the finite-difference scheme:

$$
\begin{aligned}
& \frac{\beta_{i}-\beta_{i-1}}{h}=\Phi_{i-1 / 2}, \quad \Phi_{i-1 / 2}=\Phi\left(\beta_{i-1 / 2}, \bar{r}_{i-1 / 2}, \bar{z}_{i-1 / 2}, L\right) \\
& \frac{\bar{z}_{i}-\bar{z}_{i-1}}{h}=\sin \beta_{i-1 / 2}, \quad \frac{\bar{r}_{i}-\bar{r}_{i-1}}{h}=\cos \beta_{i-1 / 2}, \quad i=\overline{1, N} \\
& \beta_{i-1 / 2}=\frac{\beta_{i-1}+\beta_{i}}{2}, \quad \bar{r}_{i-1 / 2}=\frac{\bar{r}_{i-1}+\bar{r}_{i}}{2}, \quad \bar{z}_{i-1 / 2}=\frac{\bar{z}_{i-1}+\bar{z}_{i}}{2} \\
& \beta_{0}=\alpha-\frac{\pi}{2}, \quad \beta_{N}=-\alpha, \quad \bar{r}_{0}=\frac{1}{L}, \quad \bar{z}_{N}=0, \quad L=\left(\frac{U}{I_{0}}\right)^{1 / 3}
\end{aligned}
$$

where the solution $\left(\bar{z}_{i}, \bar{r}_{i}\right)$ of the difference scheme is an approximation of the unknown solution $(\bar{z}, \bar{r})$ of the differential problem (15) at the grid nodes $\bar{s}_{i}, i=\overline{1, N}$. The integrals $I_{0}, I_{1}$ and $I_{2}$ are computed by the trapezoidal rule.

Since for $\beta_{i}$, we have the boundary conditions $\beta_{0}=\alpha-\pi / 2$ and $\beta_{N}=-\alpha$, the recursion formula could be used to compute $\beta_{i-1}$ from $\beta_{i}$ for $i=N, N-1, \ldots, 2$ or contrary $\beta_{i}$ from $\beta_{i-1}$ for $i=1,2, \ldots, N-1$. Both variants of the tangential method have been discussed in [9]; the first one turns out to be more stable and resulted in a faster iterative algorithms. Therefore, we used the first variant solving

$$
\left.\begin{array}{l}
\beta_{i}^{n+1}=\beta_{i+1}^{n+1}-h \Phi_{i+1 / 2}^{n}+(1-\tau)\left(\beta_{i}^{n}-\beta_{i+1}^{n}+h \Phi_{i+1 / 2}^{n}\right) \\
i=N-1, N-2, \ldots, 1, \quad \beta_{N}^{n+1}=-\alpha, \quad \beta_{0}^{n+1}=\alpha-\frac{\pi}{2}
\end{array}\right\}
$$

where $n=0,1,2, \ldots$ is the iteration number, $\tau>0$ is the relaxation parameter. Computations at each iteration are conducted by recurrence formulas. First, the recurrence rule (16) is used to compute the grid values of $\beta_{i}^{n+1}$. Then, by employing procedures (17) and (18), new iterative approximations are determined for the free surface coordinates. And, finally, the grid function $\Phi_{i+1 / 2}^{n+1}$ and the dimensionless length $L^{n+1}$ are formed by the found values of $\bar{r}_{i}^{n+1}, \bar{z}_{i}^{n+1}, \beta_{i}^{n+1}$.

All computations have been performed on the uniform grid with the mesh size of $h=1 / N=1 / 4000$.

\section{Numerical results}

The numerical study has been performed for fixed values $B o=1, A_{1}=6, U=400, \alpha=45^{\circ}$ and $90^{\circ}$, for a wide range of values of the parameter $A_{2}$ characterizing the magnitude of the current strength in the conductor. Note that the values for $B o, A_{1}$, and $U$ correspond the experimental data used in [6]. In order to study the influence of the diffusion process, computations have been carried out both taking the diffusion effect into consideration and assuming a uniform concentration of the particles in the fluid. In the latter case, the mathematical model described in [6] has been used.

Characteristic equilibrium axisymmetric shapes of the drop at $\alpha=90^{\circ}$ for three values of $A_{2}$ are represented in Fig. 2 . They show that increasing the parameter $A_{2}$ causes the displacement of the contact line on the plate in the direction of the magnetic field gradient, i.e. closer to the conductor, and accordingly the elevation of the contact line with the conductor becomes higher. As illustrated, the particle diffusion gives rise to appreciable strengthening of this process compared to a uniform particle concentration. In Fig. 3, plots of basic geometric characteristics of the magnetic fluid drop in dependence of the magnetic parameter $A_{2}$ are shown, where $z_{0}=z(0)$ denotes the height of the drop and $r_{1}=r(L)$ the radius of its base. We see a considerable dependence, both quantitatively and qualitatively, between the case of approximating the particle concentration as uniform and the case of taking into account the diffusion of magnetic particles. In the former case, the drop height grows almost linearly for increasing $A_{2}$ and the radius is monotonically decreasing. In the latter case, growth of the drop height is much more intensive, but of special interest is the behaviour of the drop radius $r_{1}$ for increasing $A_{2}$ : first it decreases up to some value and then it begins to increase, i.e. the fluid withdrawal from the conductor takes place. This effect can be explained by intense redistribution of the particles as the parameter $A_{2}$ grows (see Fig. 4). At sufficiently large values of $A_{2}$, a great bulk of particles are concentrated in the close vicinity of the conductor, while their concentration far from the conduc- 


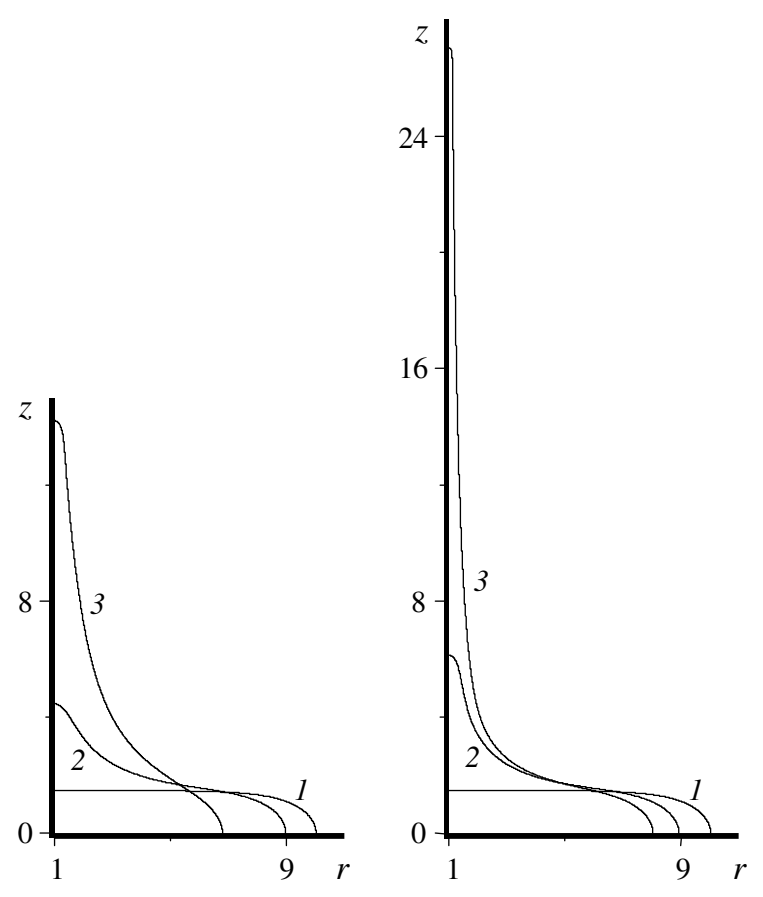

Fig. 2. Free surface shapes at $\alpha=90^{\circ}$ : (1) $A_{2}=0$, (2) $A_{2}=3$ and (3) $A_{2}=6$. Uniform particle concentration (left) and non-uniform particle concentration (right).
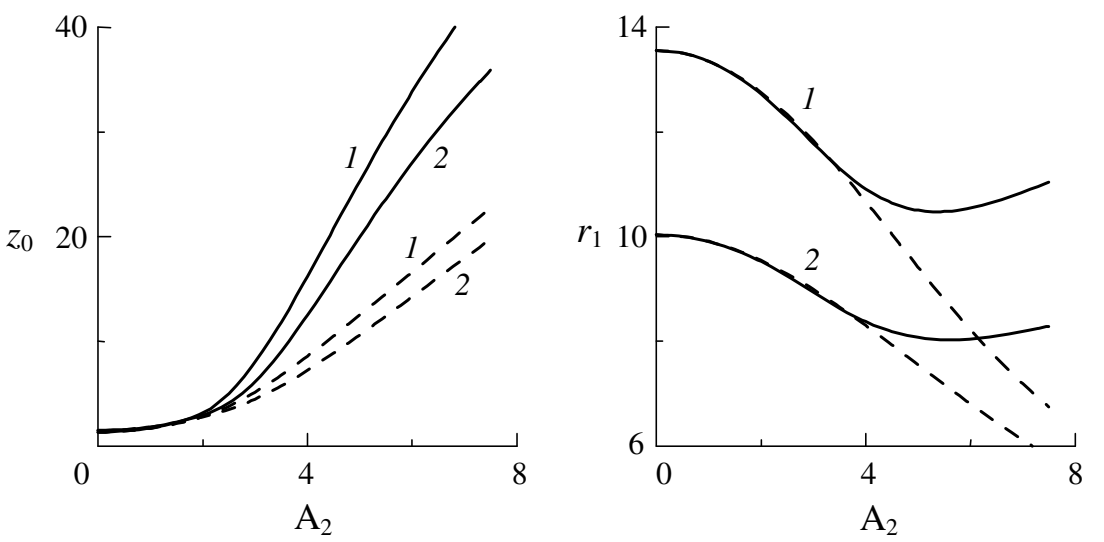

Fig. 3. Characteristic sizes of the drop depending on the parameter $A_{2}:(1) \alpha=45^{\circ} ;(2) \alpha=90^{\circ}$. Solid lines correspond to a non-uniform particle concentration, the dashed lines to a uniform approximation.

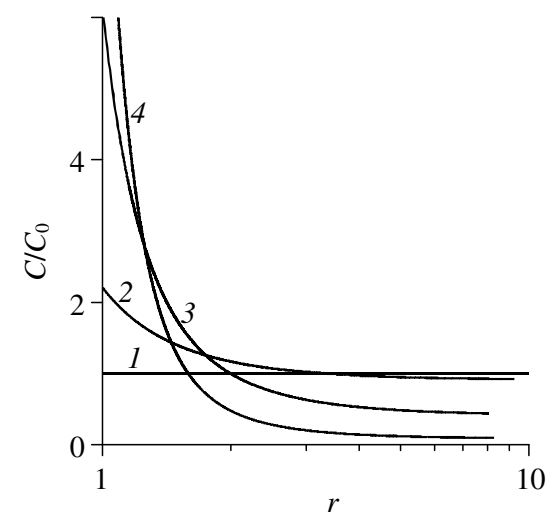

Fig. 4. Distribution of particle concentration in the magnetic fluid drop depending on parameter $A_{2}$ at $\alpha=90^{\circ}:$ (1) $A_{2}=0$, (2) $A_{2}=2.5$, (3) $A_{2}=5$ and (4) $A_{2}=7.5$. 
tor is close to zero and does not influence appreciably the free surface shape. In other words, near the contact point situated on the plate the force impelling the fluid to move in the direction of conductor is negligible and in this neighbourhood the free surface is shaped mainly under the action of capillary forces and gravity.

\section{Summary}

The numerical results illustrated in several plots give grounds for the conclusion that the diffusion of ferromagnetic particles in a magnetic fluid under the action of a non-uniform magnetic field greatly influences the free magnetic fluid surface shape. The approximation by a uniform particle concentration seems to be applicable only on ferrohydrostatics problems with nearly uniform magnetic fields.

\section{Acknowledgements}

The authors would like to thank the German Academic Exchange Service (DAAD) for partially supporting the research in this paper.

\section{References}

[1] Rosensweig RE. Ferrohydrodynamics. New York: Dover Publ. Inc.; 1997.

[2] Berkovski B, Bashtovoi V. Magnetic fluids and applications handbook. New York: Begell House Publ.; 1996.

[3] Bashtovoi VG, Berkovsky BM, Vislovich AN. Introduction to thermomechanics of magnetic fluids. Washington: Hemisphere Publ.; 1988.

[4] Berkovsky BM, Medvedev VF, Krakov MS. Magnetic fluids: engineering applications. Oxford: Oxford University Press; 1993.

[5] Blums E, Cebers A, Maiorov MM. Magnetic fluids. Berlin: Walter de Gruyter; 1997.

[6] Bashtovoi VG, Budnik AM, Polevikov VK, Reks AG. Doubly connected equilibrium configurations of ferrofluid in magnetic field of vertical conductor. Magnetohydrodynamics 1984;20(2):144-50.

[7] Bashtovoi VG, Polevikov VK, Suprun AE, Stroots AV, Beresnev SA. Influence of Brownian diffusion on the statics of magnetic fluid. Magnetohydrodynamics 2007;43(1):17-25.

[8] Polevikov V, Tobiska L. On the solution of the steady-state diffusion problem for ferromagnetic particles in a magnetic fluid. Math Model Anal 2008;13(2):233-40

[9] Polevikov VK. Methods for numerical modeling of two-dimensional capillary surfaces. Comput Method Appl Math 2004;4(1):66-93. 